\title{
Real-Time Construction Simulation Coupling a Concrete Temperature Field Interval Prediction Model with Optimized Hybrid-Kernel RVM for Arch Dams
}

\author{
Wenshuai Song, Tao Guan, Bingyu Ren *, Jia Yu, Jiajun Wang ${ }^{\circledR}$ and Binping Wu \\ State Key Laboratory of Hydraulic Engineering Simulation and Safety, Tianjin University, Tianjin 300350, China; \\ tju_song@tju.edu.cn (W.S.); tao.guan@tju.edu.cn (T.G.); yujia@tju.edu.cn (J.Y.); jiajun_2014_bs@tju.edu.cn (J.W.); \\ wubinping@tju.edu.cn (B.W.) \\ * Correspondence: renby@tju.edu.cn; Tel.: +86-022-2789-0911
}

Received: 24 July 2020; Accepted: 28 August 2020; Published: 31 August 2020

check for updates

\begin{abstract}
Joint grouting simulation is an important aspect of arch dam construction simulation. However, the current construction simulation model simplifies the temperature factors in joint grouting simulation, which leads to the difference between the simulation results and the actual construction schedule. Furthermore, the majority of existing temperature prediction research is based on deterministic point predictions, which cannot quantify the uncertainties of the prediction values. Thus, this study presents a real-time construction simulation method coupling a concrete temperature field interval prediction model to address these problems. First, a real-time construction simulation model is established. Secondly, this paper proposes a concrete temperature interval prediction method based on the hybrid-kernel relevance vector machine (HK-RVM) with the improved grasshopper optimization algorithm (IGOA). The hybrid-kernel method is adopted to ensure the prediction accuracy and generalization ability of the model. Additionally, the improved grasshopper optimization algorithm (IGOA), which utilizes the tent chaotic map and cosine adaptive method to improve the algorithm performance, is developed for the parameter optimization of HK-RVM. Thirdly, concept drift detection based on variable window technology is proposed to update the prediction model. Finally, an arch dam project in China is used as a case study, by which the superiority and applicability of the proposed method are proven.
\end{abstract}

Keywords: arch dam; construction simulation; concrete temperature field; interval prediction; relevance vector machine; grasshopper optimization algorithm; concept drift

\section{Introduction}

Arch dams have the advantages of strong overload capacity, good seismic performance, and saving construction materials, which have become the popular dam type for high dam construction in China [1]. In order to prevent temperature cracks, an arch dam is usually divided into several dam monoliths along the dam axis direction during the construction period. The surface between adjacent dam monoliths is called the transverse joint, which is where the grouting system is embedded [2]. For the sake of the dam's integrity and safety, it is essential to grout on the transverse joint surface during the construction period. Then, the independent dam body will be cemented as a whole. Therefore, joint grouting is an essential procedure in arch dam construction. In addition, if the joint grouting lags behind, the dam concrete pouring will be influenced due to the cantilever height constraint, which will lead to a delay in the overall construction progress of the dam. Therefore, joint grouting schedule management is an important aspect of dam construction schedule management, which has a crucial influence on the safety and the construction schedule of a dam project. 
With the development of computer and system simulation technology, construction simulation has become an important tool for project construction schedule analysis and management [3]. This technology enables us to realize a simulation of the dynamic process of project construction and provides scientific, reliable, and reasonable construction schedule arrangements and resource allocation schemes. Thus far, extensive research has been conducted on construction simulation models, such as discrete event simulation (DES) [4], system dynamics (SD) [5], agent-based model (ABM) [6], hybrid SD-DES [7], hybrid SD-ABM [8], and fuzzy simulation technology [9]. These simulation models lay a solid foundation for the study of arch dam construction simulations. Zhong $[10,11]$ constructed a simulation modeling framework of high arch dam construction, and put forward a real-time interactive simulation method for arch dams based on discrete event simulation (DES) and virtual reality technology. Afterwards, Zhong [12] established a real-time construction progress control model that considered temperature information and quality inspection information during the construction period. Zhou [13] also proposed a simulation model that combined the real-time temperature monitoring data and the actual construction schedule within the temperature field simulation model. Although these studies provide technical support for arch dam construction schedule analysis and management, the current construction simulation model cannot accurately simulate the joint grouting construction time. The current model simplifies the influence of the temperature field on the joint grouting. The model converts the temperature value into the concrete age based on the experience curve when the concrete temperatures meet the temperature control requirements. The experience curve of concrete internal temperature is provided by the design institute. However, the temperature field of dam concrete is complex and unstable during the construction period, and is affected by many uncertain factors (pouring sequence, pouring temperature, ambient temperature, cooling water flow, cooling water temperature, surface insulation, the heat conduction between the dam block, and so on) $[14,15]$. Therefore, the temperature change process cannot fully conform to the empirical temperature curve provided by the design institute. This results in a deviation between the simulation schedule and the actual situation. Consequently, it is necessary to study how to realize the real-time prediction of the concrete temperature field to improve simulation accuracy and ensure the effectiveness of the simulation schedule plan.

Generally speaking, the concrete temperature field is dynamic, unstable, nonlinear. Thus, it is complicated to precisely describe the temperature change process. Yet, due to the easy-to-use and flexible modeling properties, data-driven approaches have gradually become a popular method for temperature prediction [16-18]. Data-driven approaches can reflect the relationship between the influencing factors and the outputs. Generally, the prediction models are mainly divided into point prediction and interval prediction according to different prediction forms. These point prediction methods have been applied in the field of temperature prediction, including back-propagation neural network [19,20], artificial neural network [21], gray model [22], genetic algorithm [23,24], and support vector machine $[25,26]$. However, point prediction only provides one prediction result at each target point, which lacks uncertainty estimation $[27,28]$. For concrete temperature data in the construction period, due to the existence of data noise in monitored temperature data, the complex and changeable external factors, as well as the subjective uncertainty of sample selection, the point prediction method experiences difficulty in quantifying the uncertainties of the concrete temperature prediction values. The concrete temperature prediction value is an important input parameter for joint grouting simulation. The uncertainty of concrete temperature prediction will lead to inaccuracy in the simulation results and affect the reliability of the results. On the other hand, interval prediction can provide the prediction intervals at a certain confidence level, and this can quantify the uncertainty of the prediction value [29-31]. Traditional methods for the construction of prediction intervals include Bayesian [32-34] and bootstrap [35-37]. However, these prediction interval methods are constructed based on the point prediction error and require massive computational requirements.

With the advantages of high sparse, simple parameters, flexible kernel selection, strong generalization ability, and good probability prediction ability, relevance vector machine (RVM) 
is an effective approach for interval prediction, and has been widely used [38-40]. RVM is based on a probabilistic Bayesian learning framework, which can provide probabilistic predictions. Besides, the prediction effect of the RVM model depends on the selection of the kernel functions and the kernel parameters [41]. A single kernel function cannot have a strong learning ability and generalization ability at the same time. Hybrid-kernel learning is a good solution [42]. Based on specific rules, multiple single kernel functions are combined into a new kernel function. For the problem of parameter optimization, intelligent optimization algorithms are introduced to determine the optimal parameters. Many scholars adopted different intelligent optimization algorithms to optimize the parameters of RVM, including the ant lion optimizer [43], whale optimization algorithm [44], imperialist competitive algorithm [45], cuckoo search optimization [46], particle swarm optimization [47], gravitational search optimization [48], and quantum-inspired gravitational search [49]. The grasshopper optimization algorithm (GOA), proposed by Saremi, is a new swarm intelligence algorithm [50]. The basic idea was inspired by the swarm behavior of grasshoppers. This algorithm has the advantages of simple structure, strong stability, fewer parameters, and faster convergence, which has been widely used in parameter optimization [51].

The temperature data in the construction period are a group of dynamic flow data that grows rapidly over time, and the environmental temperature and water-cooling stage transition in the dam construction period change with time. These data characteristics may cause the prediction performance of the prediction model to decline significantly over time. This is called concept drift. The study of concept drift detection methods for the prediction model is necessary to realize the dynamic updating of the prediction model. For concept drift detection methods, window-based detection methods are effective [52,53], such as efficient concept-adapting very fast decision tree (E-CVFDT) [54] and Adwin [55]. The window size of these methods is sensitive to concept drift detection. A fixed window can improve the analysis speed; however, it is difficult to determine the size of the window. In addition, it is not easy to recognize the concept drift when the window size is too large, and it is difficult to update the model when the window size is too small due to the small amount of data. In order to deal with this problem, this paper proposes a concept drift detection method based on the variable window technique.

In summary, the current construction simulation studies simplified the influence of temperature factors on joint grouting. Additionally, the majority of existing temperature prediction research is based on point prediction, which cannot effectively quantify the uncertainty of the concrete temperature prediction value. Therefore, the aim of this research was to propose a real-time construction simulation method coupling a concrete temperature interval prediction model with the improved grasshopper optimization algorithm. The main aim of this paper is to:

1. Establish a real-time construction simulation model that couples the concrete temperature field to realize a more accurate simulation.

2. Propose a concrete temperature interval prediction model based on the hybrid-kernel relevance vector machine (HK-RVM) and the improved grasshopper optimization algorithm (IGOA) to obtain reliable prediction intervals of concrete temperature.

3. Develop a concept drift detection method based on the variable window technology to update the prediction model.

The remainder of this paper is organized as follows: The framework of this paper is shown in Section 2. In Section 3, a detailed description of the proposed methodologies is presented. In Section 4, we present a case study to verify the applicability of the proposed method. In Section 5 , we provide a discussion to investigate the superiority of the proposed method. Section 6 outlines the conclusions and future works. 


\section{Research Framework}

In this work, a real-time construction simulation method coupling the concrete temperature field interval prediction model was proposed. The research framework is composed of three levels: the construction simulation model, the temperature interval prediction model, and a case study, as shown in Figure 1.

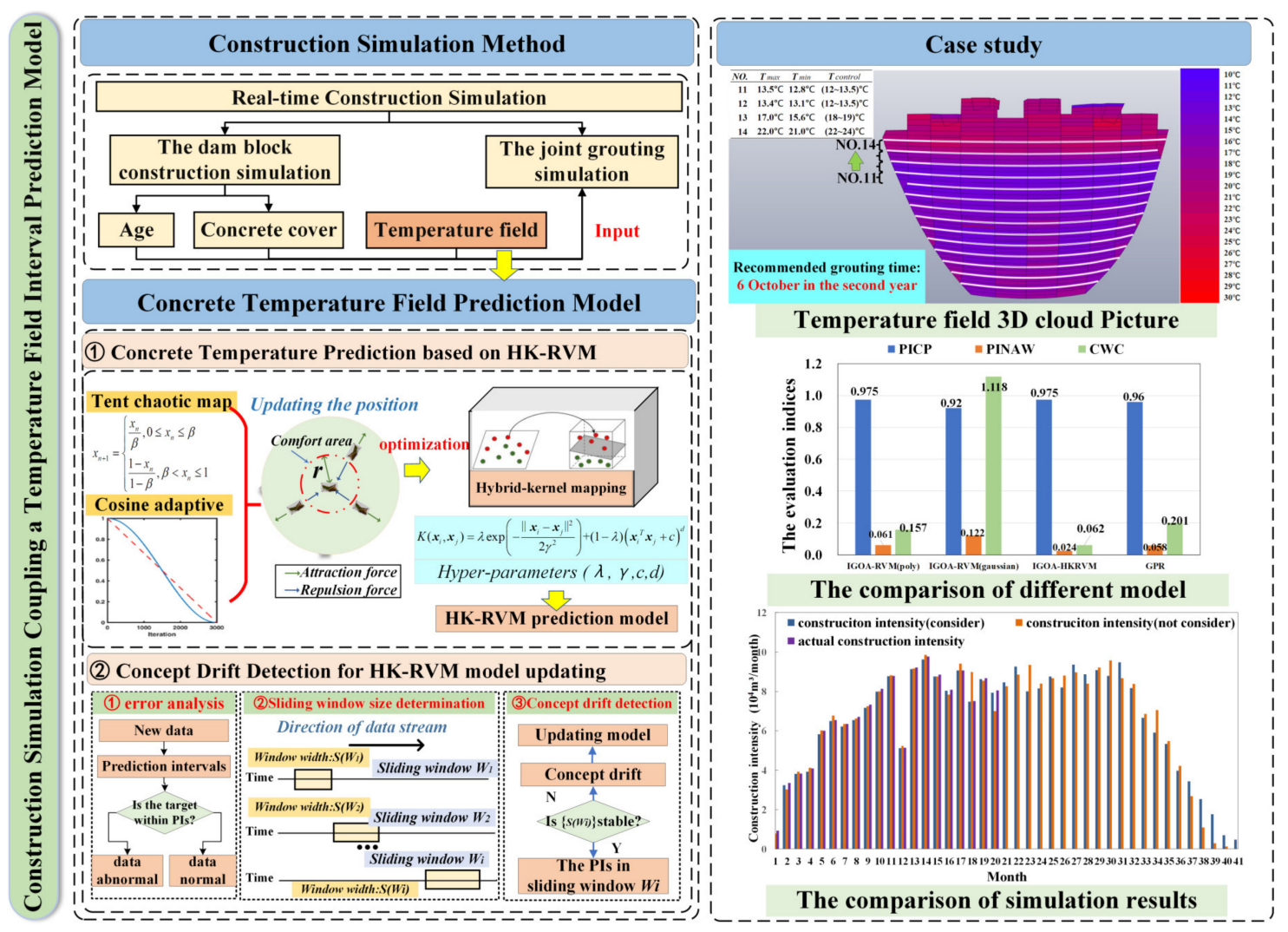

Figure 1. Research framework.

At the construction simulation model level, the real-time construction simulation model was divided into the dam block construction simulation and the joint grouting simulation. The concrete age, cover weight, and temperature field are the key parameters for the joint grouting simulation, and these were obtained from the dam block construction simulation results. The temperature field can be predicted based on the interval prediction model.

At the concrete temperature field prediction model level, it contains the concrete temperature interval prediction model and the concept drift detection for model updating. The details are as follows: (1) In the sublevel of temperature interval prediction model, firstly, the tent chaotic map and cosine adaptive methods are adopted to improve the performance of the grasshopper optimization algorithm (GOA). Secondly, the IGOA is used for parameter optimization of the HK-RVM prediction model. (2) In the sublevel of concept drift detection for model updating, a concept drift detection based on the variable window technique is proposed to update the prediction model to avoid prediction performance degradation. Firstly, the error of new data is analyzed based on the established temperature interval prediction model. Secondly, the sliding window size is determined according to the error analysis results. Thirdly, whether concept drift occurs depends on the change of the sliding window size. If the concept drift occurs, the temperature interval prediction model will be retrained. Finally, a dynamic concrete temperature interval prediction model can be established.

At the case study level, the proposed model was applied to an arch dam in China. The temperature field prediction results and the construction simulation scheme were obtained. Compared with 
the relevance vector machine (RVM) model with a single kernel function and Gaussian process regression (GPR) model, the prediction accuracy and generalization ability of the proposed model were verified. Compared with the traditional simulation model without considering the temperature field, the simulation results with a considerable temperature field were closer to the actual situation.

\section{Methodology}

\subsection{Problem Formulation}

According to the construction method of arch dams, the construction simulation of arch dams can be simplified into two parts: the dam block construction simulation and joint grouting simulation. According to the requirements of construction specifications, the joint grouting should consider not only the concrete internal temperature in the temperature control area during the construction period but also the concrete age and cover weight. The concrete age is related to the pouring time of the dam block, and the concrete cover weight is related to the current dam pouring face, and these are all obtained by the dam block simulation. The concrete temperature field is predicted by the HK-RVM interval prediction model. In addition, dam construction progress and joint grouting progress are mutually restricted due to the cantilever height constraint. The real-time construction simulation mathematical model is expressed in Figure 2. The model defines the objective function of real-time construction simulation, dam block construction simulation, joint grouting simulation, and the relationship between dam block construction simulation and joint grouting simulation. The detailed descriptions of the symbols are shown in Appendix A.

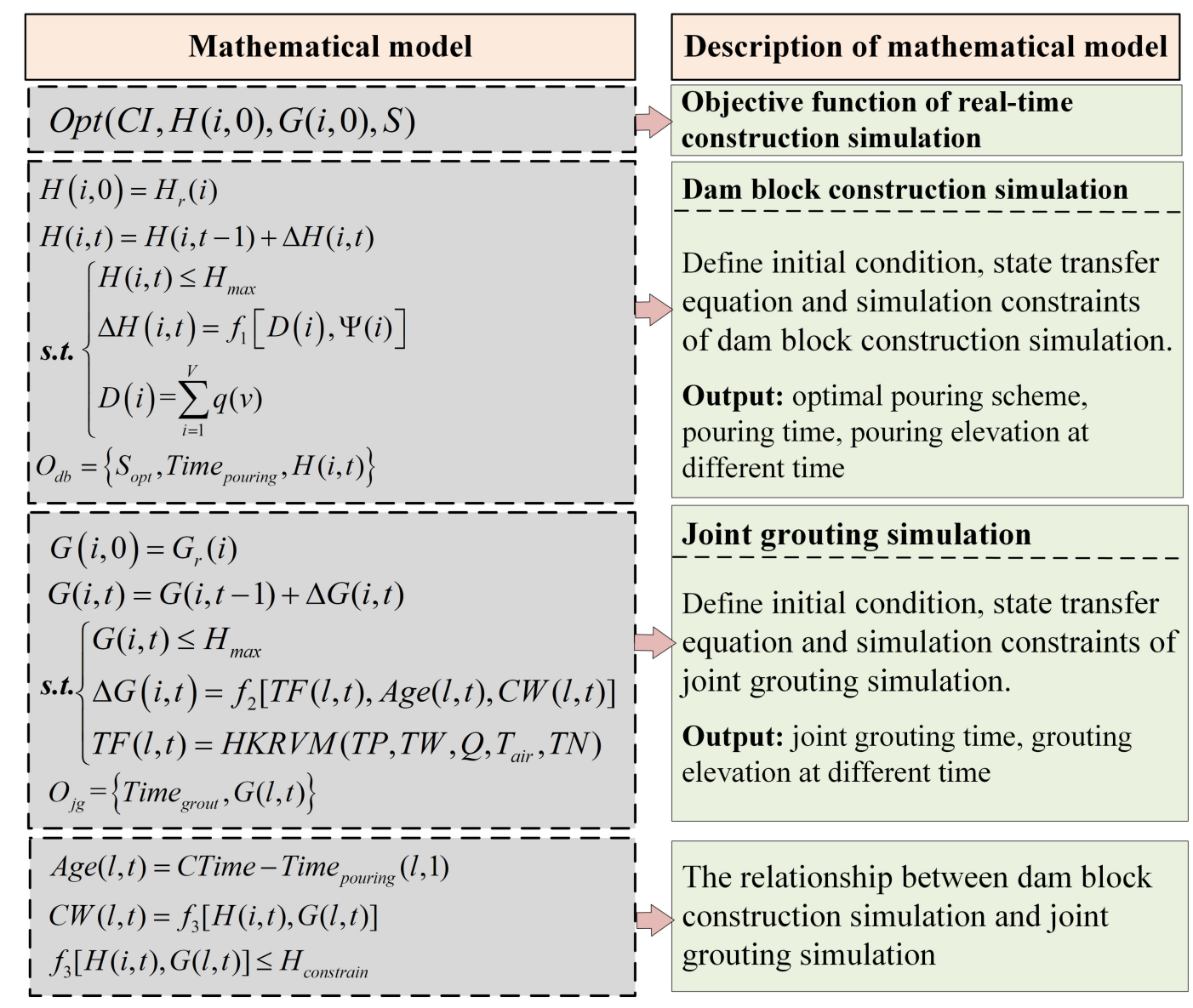

Figure 2. The real-time construction simulation mathematical model. 


\subsection{Concrete Temperature Field Prediction Model}

\subsubsection{Hybrid-Kernel Relevance Vector Machine}

Given a training sample set $\left\{x_{i}, y_{i}\right\}_{i=1}^{N}\left(x_{i} \in R^{d}, y_{i} \in R\right)$, the relevance vector machine model can be defined as follows:

$$
y_{i}=\sum_{i=1}^{N} w_{i} K\left(x, x_{i}\right)+w_{0}+\varepsilon
$$

where $N$ is the number of data samples, $w_{i}$ are the model weights, $\varepsilon \sim N\left(0, \sigma^{2}\right)$ is the independent additive Gaussian noise, and $K\left(x, x_{i}\right)$ is a nonlinear kernel function.

The selection of the kernel function is the key to the model performance. Among many kernel functions, the polynomial kernel function is a typical global kernel function with a strong generalization ability [42]. The gauss kernel function is a type of local kernel function with a good local learning ability [42]. The concrete temperature data are dynamic and nonlinear. The concrete temperature is influenced by the continuous change of the hydration heat, as well as the random fluctuation of the external conditions. A single kernel function cannot meet the temperature prediction demand [42]. In order to ensure the global generalization ability and local learning ability of the model, the hybrid-kernel method is employed in this paper. The expression is shown as follows:

$$
K\left(x_{i}, x_{j}\right)=\lambda \exp \left(-\frac{\left\|x_{i}-x_{j}\right\|^{2}}{2 \gamma^{2}}\right)+(1-\lambda)\left(x_{i}^{T} x_{j}+c\right)^{d}
$$

where the first term on the right side of the equation is the gauss kernel function, $\gamma$ is the kernel parameter of the gauss kernel function. The second term is the polynomial kernel function, $c$ and $d$ are the kernel parameters of the polynomial kernel function. $\lambda$ is the adjustment coefficient.

Suppose the target value $y_{i}$ is independent, then the likelihood function can be described as

$$
p\left(y \mid w, \sigma^{2}\right)=\prod_{i=1}^{N} N\left(\Phi w, \sigma^{2}\right)
$$

where $w=\left(w_{0}, w_{1}, \ldots, w_{N}\right)^{T} ; \Phi=\left(\phi_{1}, \phi_{2}, \ldots, \phi_{N}\right)^{T} ; \phi_{i}=\left(1, K\left(x_{i}, x_{1}\right), \ldots, K\left(x_{i}, x_{N}\right)\right)$

According to the Bayesian criterion and multivariate conditional Gaussian distribution, the posterior distribution can be written as

$$
p\left(w \mid \alpha, \sigma^{2}, y\right)=\frac{p\left(y \mid w, \sigma^{2}\right) p(w \mid \alpha)}{p\left(y \mid \alpha, \sigma^{2}\right)}=\prod_{i=0}^{N} N(\mu, H)
$$

where $\mu=\sigma^{-2} H \Phi^{T} I y, H=\left(\sigma^{-2} \Phi^{T} I \Phi+A\right)^{-1}$ and $A=\operatorname{diag}\left(a_{0}, a_{1}, \ldots, a_{N}\right)$.

At last, the hyperparameter $\alpha$ and the variance $\sigma^{2}$ can be estimated using the maximum likelihood method. For new data $x^{*}$, the prediction value $y^{*}$ and variance $\sigma_{*}^{2}$ can be described as follows:

$$
\left\{\begin{array}{l}
y^{*}=\mu^{T} \Phi\left(x^{*}\right) \\
\sigma_{*}^{2}\left(x^{*}\right)=\left(\sigma^{2}\right)_{b e s t}+\Phi\left(x^{*}\right)^{T} H \Phi\left(x^{*}\right)
\end{array}\right.
$$

Given the $1-\zeta$ confidence level $(\mathrm{CI})$, the confidence interval of prediction value $y^{*}$ can be described as

$$
\left(L_{b}{ }^{\xi}, U_{b}^{\xi}\right)=\left(y^{*}-z_{\xi / 2} \sigma_{*}^{2}, y^{*}+z_{\xi / 2} \sigma_{*}^{2}\right)
$$

where $L_{b}^{\xi}$ is the lower bound of the prediction intervals, and $U_{b}^{\xi}$ is the upper bound of the prediction intervals. $z_{\xi / 2}$ is the bilateral quantiles of a standard normal distribution. 
The prediction accuracy of the HK-RVM model depends on the selection of kernel function parameters. According to the HK-RVM model, the parameters involved can be expressed as $(\lambda, \gamma, c, d)$. In the next section, the IGOA algorithm will be introduced to optimize the model parameters.

\subsubsection{Improved Grasshopper Optimization Algorithm (IGOA)}

The grasshopper optimization algorithm (GOA) is a new metaheuristic optimization algorithm [50]. The GOA mimics the swarming behavior of grasshoppers. The grasshoppers are affected by social interaction, gravity and wind advection in the process of migration and foraging. For the optimization problem, the influence of gravity is usually ignored, and the wind direction is assumed to always point to the target position. Then, the mathematical model can be represented as follow:

$$
X_{i}^{d}=c\left\{\sum_{\substack{j=1 \\ j \neq i}}^{N} c \frac{u b_{d}-l b_{d}}{2} s\left(\left|x_{j}^{d}-x_{i}^{d}\right|\right) \frac{x_{j}-x_{i}}{\left|x_{j}-x_{i}\right|}\right\}+\widehat{T}_{d}
$$

where $c$ is a decreasing coefficient, $u b_{d}$ and $l b_{d}$, respectively, represent the upper bound and lower bound in the $d$ th dimension, $\widehat{T}_{d}$ is the best solution so far in the $d$ th dimension, and $s$ is a function to define the strength of social forces, as shown in Equation (8).

$$
s(r)=f e^{\frac{-r}{l}}-e^{-r}
$$

where $f$ denotes the attraction intensity between grasshoppers, $l$ is the length scale of attraction, and $r$ is the range of comfortable areas. The force between grasshoppers can be divided into attraction and repulsion according to the strength of the social forces.

In the standard GOA algorithm, the control parameter $c$ usually adopts the linear method to dynamically adjust. It means that the parameter $c$ decreases at a constant rate. In order to effectively balance the exploration and exploitation ability of the algorithm, the cosine adaptive method is introduced to dynamically adjust parameter $c$. In the early stage, the parameter $c$ decreases more slowly than the decline rate with the linear method, which can expand the search space and increase the global searching ability. With the increase of iteration times, grasshoppers will converge near the target position. The parameter $c$ decreases more quickly than the decline rate with the linear method in the middle stage. In the later stage, the parameter $c$ decreases more slowly than the decline rate with the linear method, which can reduce the search space and increase the local exploration ability. The expression is shown as follows:

$$
c=\left\{\frac{\left(\cos \left(\frac{\pi k}{L}\right)+c_{\max }\right) \times\left(c_{\max }+c_{\min }\right)}{2}\right\}
$$

where $L$ shows the maximum number of iterations, $l$ is the current iteration, $c_{\max }=1, c_{\min }=10^{-5}$.

In order to avoid the algorithm falling into the local optimal solution, the skew tent chaotic mapping was adopted to improve the search efficiency of GOA. Compared with the logistic chaotic map, the chaos sequence generated by the skew tent chaotic map had better randomness, more uniform distribution, and was not sensitive to the initial value. This map can effectively improve the search space of GOA and accelerate the convergence of the algorithm. The expression is shown as follows:

$$
x_{n+1}=\left\{\begin{array}{l}
\frac{x_{n}}{\beta}, 0 \leq x_{n} \leq \beta \\
\frac{1-x_{n}}{1-\beta}, \beta<x_{n} \leq 1
\end{array}\right.
$$


where $x_{n}$ is the $n$th number in the sequence, $\beta$ is the control parameter of the chaotic map.

In order to show the performance of improved grasshopper optimization algorithm (IGOA), the statistical analysis of improved grasshopper optimization algorithm (IGOA) was tested on 6 unimodal benchmark functions and 4 multimodal benchmark functions. The benchmark functions used are available in Appendix B. The unimodal functions (F1-F6) have only one optimal solution, so the convergence speed and exploitation ability of the improved grasshopper optimization algorithm (IGOA) can be tested. The multimodal functions (F7-F10) have multiple local optimal solutions, so we can test the local optimization and exploration ability of the improved grasshopper optimization algorithm (IGOA) using the multimodal functions.

For solving the test functions, 50 search agents and 500 iterations were employed. Firstly, the convergence rate and global optimization ability are tested on the unimodal functions (F2) and the multimodal functions function (F7). Figure $3 a-c$ shows the function graph, respectively. Figure $3 b-d$ shows the comparison of the convergence curve. The results show that IGOA has faster convergence rates and strong global optimization ability. Considering the randomness of the algorithm, it is necessary to verify the repeatability of the IGOA. Then, each of the benchmark functions (F1-F10) was calculated 50 times. The statistical analysis results of benchmark functions (F1-F10) are provided in Table 1. The IGOA is obviously superior to GOA in both unimodal and multimodal function optimization problems.

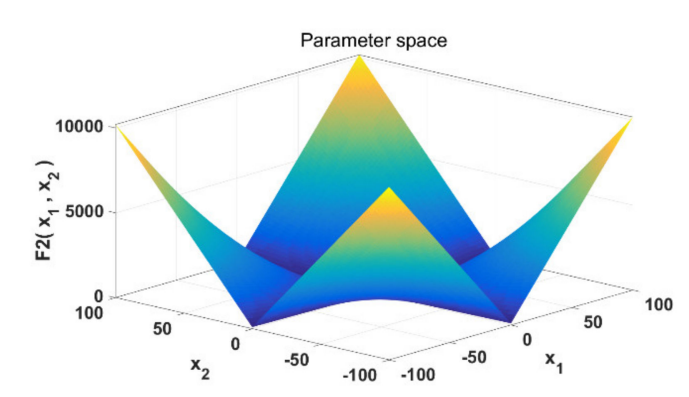

(a) The function graph of benchmark function F2

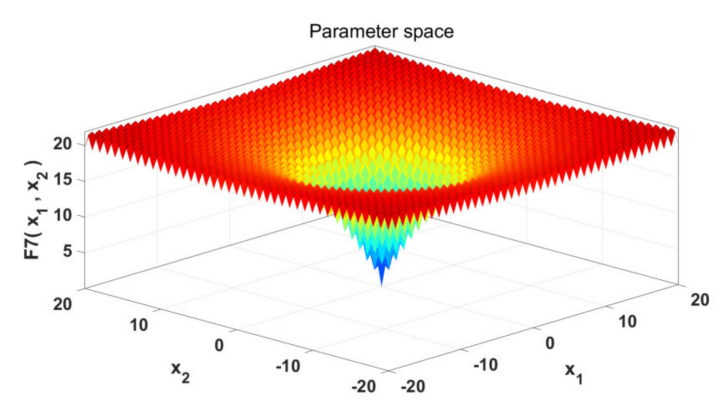

(c) The function graph of benchmark function F7

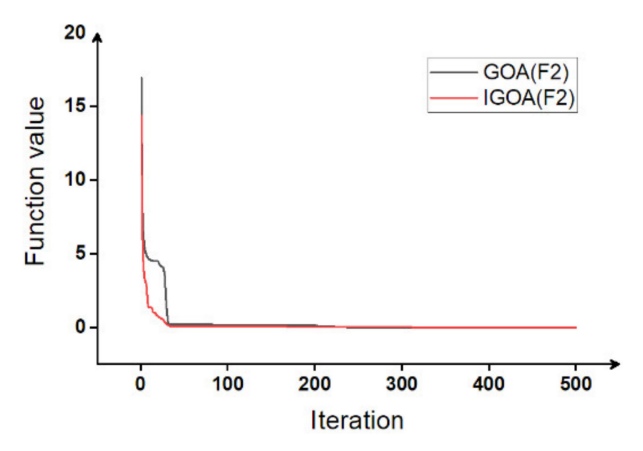

(b) The convergence curve of function F2

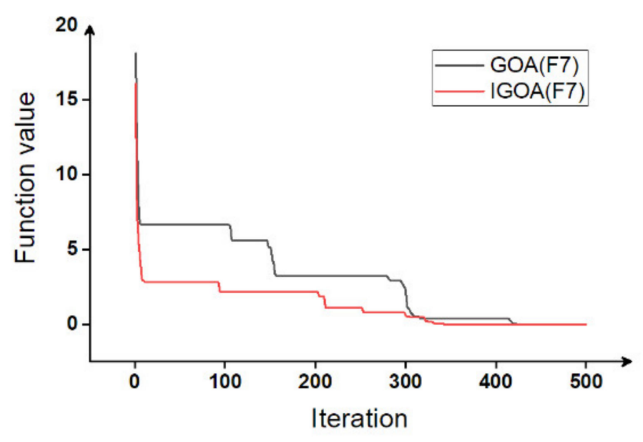

(d) The convergence curve of function F7

Figure 3. The performance comparison of improved grasshopper optimization algorithm (IGOA) and GOA on benchmark functions (F2 and F7). 
Table 1. The statistical analysis results of benchmark functions.

\begin{tabular}{ccccc}
\hline \multirow{2}{*}{ Functions } & \multicolumn{2}{c}{ IGOA } & \multicolumn{2}{c}{ GOA } \\
\cline { 2 - 5 } & Mean & Standard & Mean & Standard \\
\hline F1 & 0.0455 & 0.0005 & 0.0725 & 0.0009 \\
F2 & 0.0007 & 0.0000 & 0.0004 & 0.0000 \\
F3 & 0.0000 & 0.0000 & 0.0000 & 0.0000 \\
F4 & 0.0000 & 0.0000 & 0.0001 & 0.0000 \\
F5 & 0.0000 & 0.0000 & 0.0000 & 0.0000 \\
F6 & 0.1049 & 0.0180 & 0.1582 & 0.0512 \\
F7 & 0.0593 & 0.0098 & 0.0659 & 0.1041 \\
F8 & 0.0000 & 0.0000 & 0.0124 & 0.0076 \\
F9 & 0.0000 & 0.0000 & 0.0000 & 0.0000 \\
F10 & 0.9980 & 0.0000 & 0.9980 & 0.0000 \\
\hline
\end{tabular}

\subsubsection{Concept Drift Detection for Model Updating}

For the concrete temperature data stream, the data arrive one by one, and each temperature data point is independent of each other. We can obtain the prediction mean value and variance for each data point by the HK-RVM prediction model. According to the normal distribution characteristics, if the target value exceeds the prediction intervals at the specified confidence level, then the new monitoring data is considered unsuitable for the current model.

As mentioned before, the window size is essential for concept drift detection. In the variable window technique, the size of the sliding window is determined by the number of target values exceeding the prediction interval at a specified confidence level. When the number reaches the specified threshold, the sliding window is closed. Then, the size of the data in the window is taken as the width of the sliding window. If there is no concept drift in the data stream, the width of the sliding window will remain stable. Therefore, we can judge whether concept drift occurs by observing the size of the sliding window. In practical applications, when concept drift is detected, the HK-RVM model will be retrained using the data in the sliding window, and then the model is updated.

\section{Case Study}

In this section, the proposed method in this paper is applied to an arch dam in southwestern China. The dam is a concrete double curvature arch dam. The maximum dam height is $270.0 \mathrm{~m}$, and the total concrete volume is 2.80 million $\mathrm{m}^{3}$. The dam is divided into 15 dam monoliths with 14 transverse joints horizontally. The dam has 29 grouting areas vertically. Joint grouting is arranged at the transverse joints between dam monoliths. In this section, we describe the historical temperature data, establish the interval prediction model of the temperature field, and calculate the simulation construction schedule coupling temperature field interval prediction model.

\subsection{Temperature Interval Prediction Model}

\subsubsection{Temperature Data Description}

By integrating the meteorological, water cooling, and thermometer data, the sample dataset was obtained. The integrated data include the environmental factors (air temperature), water-cooling factors (water temperature and water flow), heat conduction factor (concrete age, and upper and lower dam block concrete temperatures), as well as the target temperature value (dam block concrete temperature value). We collected about 4800 pieces of data in a dam block. Then, 200 pieces of data were selected as the sample dataset to establish the model, and the rest data were chosen as the dataset of concept drift detection. The model establishment is shown in Section 4.1.2. 


\subsubsection{Model Establishment}

The IGOA optimized HK-RVM was adopted to establish the concrete temperature interval prediction model in this section. The dataset was divided into a training dataset and a testing dataset using a five-fold cross-validation. Then, $20 \%$ of the data were used as the testing dataset, and the remaining $80 \%$ were used as the training dataset. For the parameters involved, the control parameter of tent chaotic map was set as 0.5 , the population number of IGOA was set as 20 , and the maximum number of iterations was set as 200 , the attraction intensity between grasshoppers was set as 0.5 , and the length scale of attraction was set as 1.5.

The convergence curves for the five models are shown in Figure 4 . The majority of the modeling converged smoothly around 40-50 iterations. The fitness values of each model were almost the same when the model converged. Then, the temperature prediction intervals model was established. The prediction intervals are shown in Figure 5 . The target values are basically within the prediction intervals at a 95\% confidence level. The model performance (prediction accuracy and generation ability) will be stated in the Discussion section.

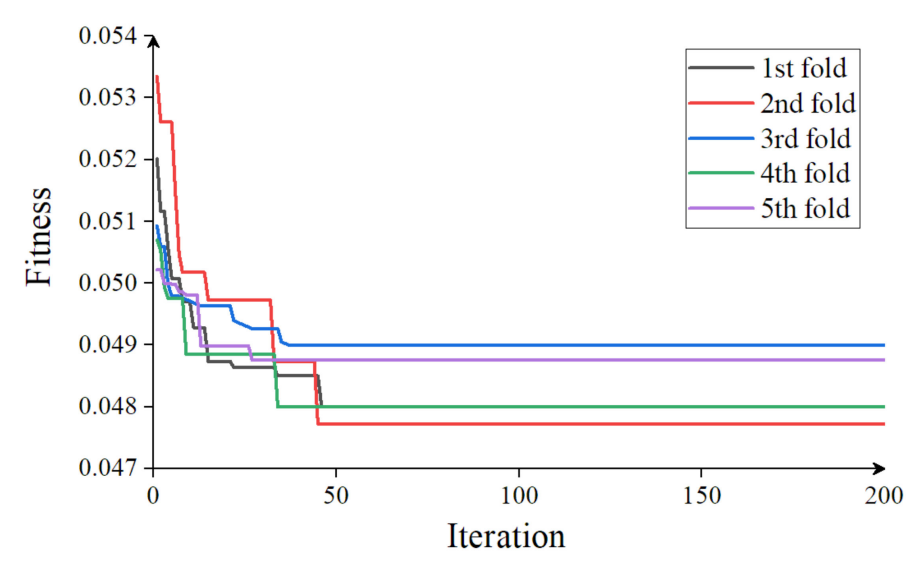

Figure 4. The convergence curves for the five-fold cross-validation.

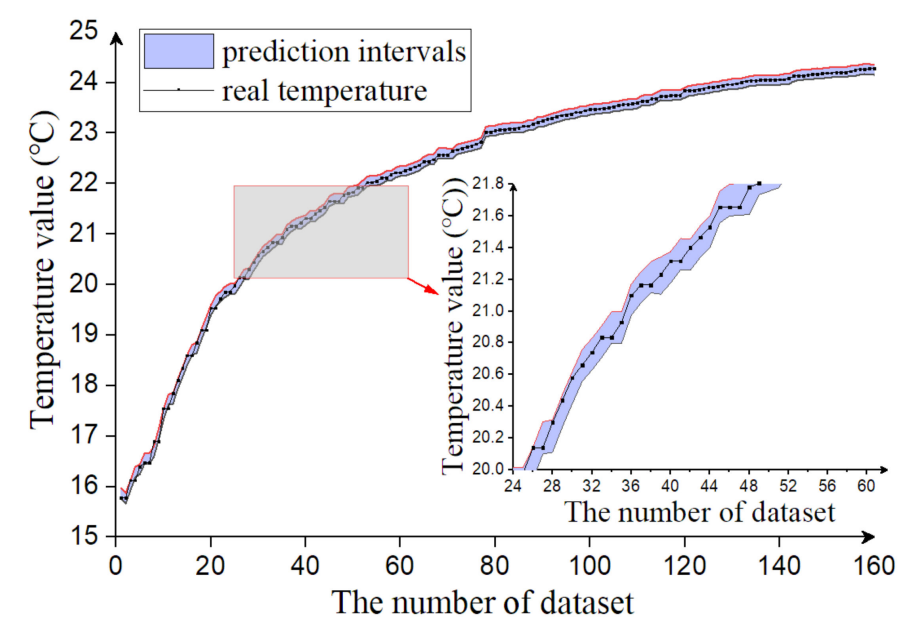

Figure 5. The temperature prediction intervals at a 95\% confidence level.

With the progress of the project, the temperature data are increasing quickly, which may exist concept drift. This will lead to the decline of the model prediction performance. Here, 4800 pieces of temperature data were collected, and then these data would be input one by one into the established HK-RVM model for concept drift detection. As mentioned in Section 3.2.3, we determine whether concept drift occurs by observing the size of the sliding window. Here, the basic window size was set as 200, and the specified threshold (the number of target values exceeding the prediction interval) was 
set as 10. Then, the size of the sliding window would be obtained, as shown in Figure 6. The size of each window fluctuated around 200, but the size of the 6th and 12th windows changed significantly. It shows that there is a sudden concept drift and the model needs to be retrained.

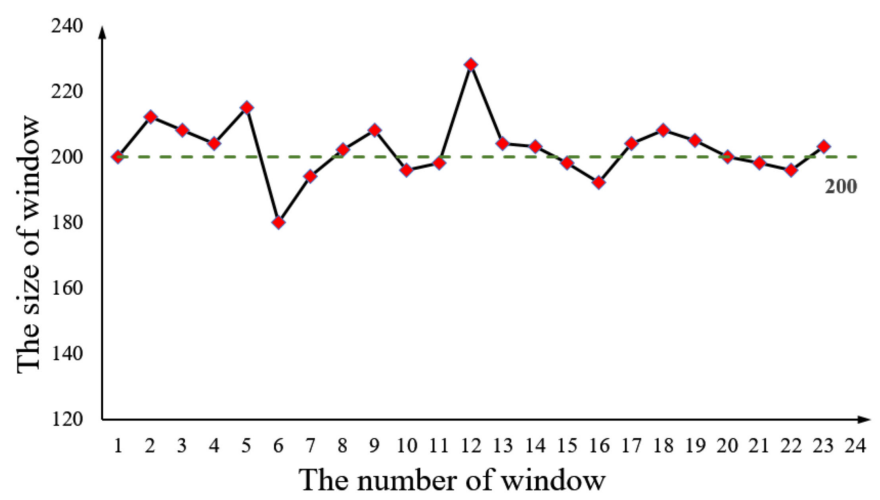

Figure 6. The curve of the sliding window size.

\subsection{Construction Simulation Coupling Temperature Field Interval Predictions}

In the real-time construction simulation mathematical model, the joint grouting simulation included three important parameters: the concrete age, the cover weight, and the temperature field. The concrete age and cover weight can be obtained using a dam block construction simulation. The temperature field is predicted by HK-RVM, as described in Section 4.1. According to the construction technical requirements, the concrete age in the grouting area should be more than 120 days old, and the cover weight of the grouting area should be more than $27.0 \mathrm{~m}$. Besides, a cooling area, a transitional cooling area, and a covering area must be set above the grouting area. Generally, the water-cooling process is divided into the first cooling stage, the midterm cooling stage, and the second cooling stage. When joint grouting, the temperature of the grouting area and the cooling area should reach the target temperature of the second cooling stage (also called closure temperature), and the temperature of the transition area should reach the target temperature of the midterm cooling stage. In this case study, the target temperature of the first cooling stage was $22-24^{\circ} \mathrm{C}$, the target temperature of the midterm cooling stage was $18-19^{\circ} \mathrm{C}$, and the target temperature of the second cooling stage was $12-13.5^{\circ} \mathrm{C}$.

Based on the established interval prediction model in Section 4.1, it was easy to obtain the temperature change curve of all dam blocks in the grouting area, and then the temperature field at a certain time can be obtained. The concrete temperature filed 3D cloud picture is shown in Figure 7 According to the temperature field results and joint grouting requirements, the recommended grouting time is 6 October in the second year.

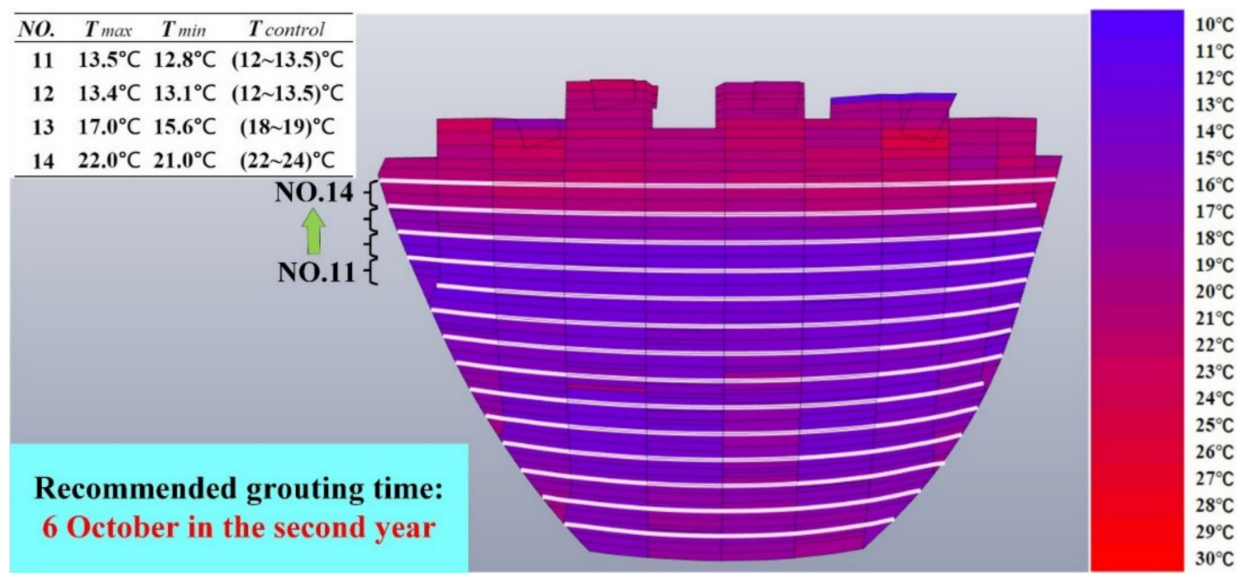

Figure 7. The 3D cloud picture of the temperature field and the recommended time for joint grouting. 


\section{Discussion}

\subsection{Prediction Model Comparison}

In this section, the prediction accuracy and generalization ability of the model were evaluated to illustrate the superiority of the proposed model. To illustrate, the training dataset is used to measure the model prediction accuracy, while the testing dataset is used to test the generalization ability. Then, the RVM model with different kernel functions (polynomial kernel, gauss kernel, and hybrid-kernel), and Gaussian process regression (GPR) were employed to compare the model performance. Three measurements were employed to compare the model performance: prediction interval coverage probability (PICP), prediction intervals normalized average width (PINAW), and coverage width-based criterion (CWC). The PICP represents the probability of the target value falling into the constructed confidence interval. The PINAW is applied to measure the width degree of the prediction interval. Generally speaking, the larger the PICP is, the more reliable the prediction interval is. The smaller PINAW is, the more accurate the prediction interval is. However, the reliability and accuracy of the model cannot be satisfied at the same time, so PICP and PINAW are contradictory indexes. The CWC can consider comprehensively the reliability and accuracy of prediction intervals. When the CWC is smaller, the prediction interval performs better in reliability and accuracy. The formulas for these measurements are presented in [56]. A detailed discussion is presented below.

- $\quad$ Model prediction accuracy

The prediction accuracy comparison of different interval prediction models was analyzed, as shown in Table 2. According to the comparison results, the PICP of different prediction models has little difference. The PINAW of IGOA-HKRVM is the smallest among the models, thus a narrower prediction range can be obtained. The CWC of IGOA-HKRVM is the smallest among all models. It reveals that the models proposed have higher coverage probability and narrower prediction intervals width than other models. Therefore, the superiority of the model prediction accuracy was proved.

Table 2. The accuracy performance of different models.

\begin{tabular}{|c|c|c|c|c|c|}
\hline Measurements & Models & IGOA-RVM $_{\text {poly }}$ & IGOA-RVM ${ }_{\text {gauss }}$ & IGOA-HKRVM & GPR \\
\hline PICP & & 0.938 & 0.942 & 0.944 & 0.942 \\
\hline PINAW & & 0.061 & 0.032 & 0.023 & 0.042 \\
\hline CWC & & 0.351 & 0.167 & 0.114 & 0.219 \\
\hline
\end{tabular}

Notes: RVM $_{\text {poly }}$ is the RVM with polynomial kernel, RVM gauss is the SVR with gauss kernel, GPR is the Gaussian process regression.

- Model generalization ability

The generalization ability comparison of different interval prediction models was analyzed, as shown in Figure 8. According to the comparison results, the PICP, PINAW, and CWC values of IGOA-HKRVM are $0.975,0.024$, and 0.062 , which are the best solution among the models. In addition, IGOA-RVM $M_{\text {gauss }}$ model performs well in the training set, but it is inferior in the testing set. However,

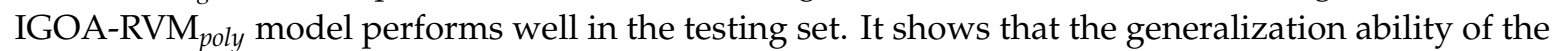
polynomial kernel function is better than the Gaussian kernel function, but the learning ability of the polynomial kernel function is inferior to the Gaussian kernel function. The hybrid-kernel method can effectively combine the advantages of polynomial kernel function and Gaussian kernel function, and the comparison results also indicate the superiority of the model generalization ability. 


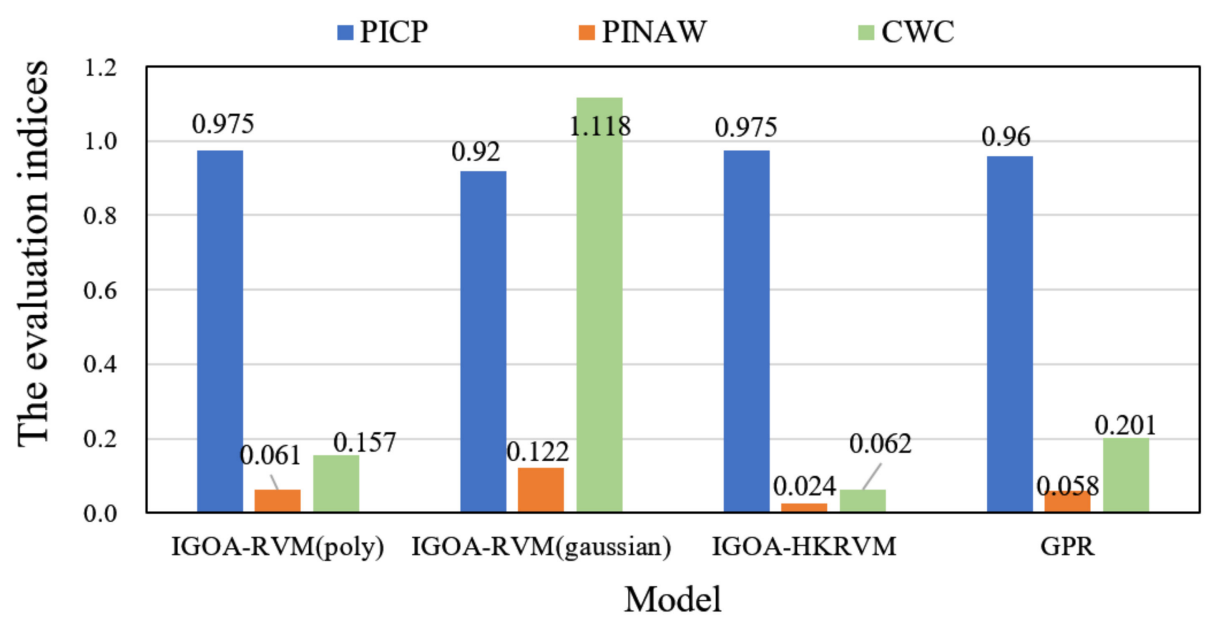

Figure 8. The comparison of different interval prediction models.

\subsection{Simulation Results Comparison}

The traditional simulation method ignores the influence of the temperature field on the joint grouting. In order to prove the advancement and applicability of the proposed method, we set the simulation results without considering the temperature field as the comparison scheme. Firstly, the joint grouting time comparison between the proposed method and the comparison scheme is carried out. The comparison between the joint grouting time with and without the temperature field and the actual grouting time is shown in Figure 9. The vertical coordinate shows the joint grouting time for each grouting area (take 15 March in the first year as time 0 ). According to the simulation results of the 1st to 11th grouting area, the joint grouting progress lags behind the comparison scheme, but is closely in accordance with the actual grouting time. It shows that the joint grouting simulation considering the temperature field can obviously improve the simulation accuracy. Based on the construction simulation model and established temperature field prediction model, the grouting time of the other grouting areas can be obtained. It can provide guidance for the subsequent dam joint grouting construction. Then, the dam construction schedule comparison between the proposed method and the comparison scheme is carried out. According to the simulation results, the dam will be completed in July in the fourth year when the temperature field is considered, and the dam will be completed in June in the fourth year when the temperature field is not considered. The dam monthly construction intensity can also be obtained by construction simulation, which can provide a reference for the production and allocation of concrete resources. The comparison of the construction intensity with and without the temperature field is shown in Figure 10.

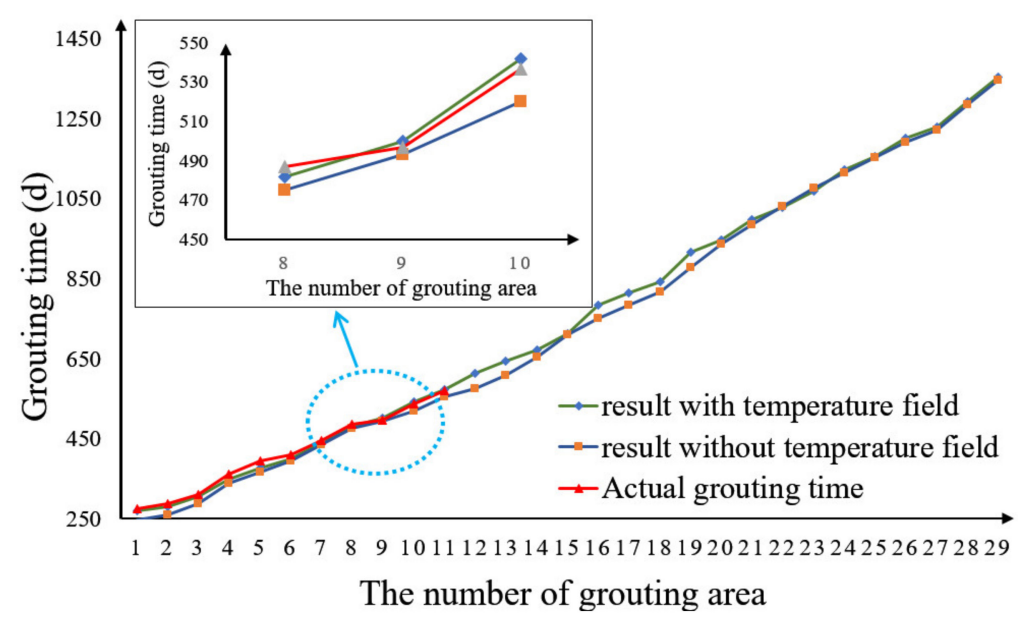

Figure 9. The comparison of the joint grouting time with and without the temperature field. 


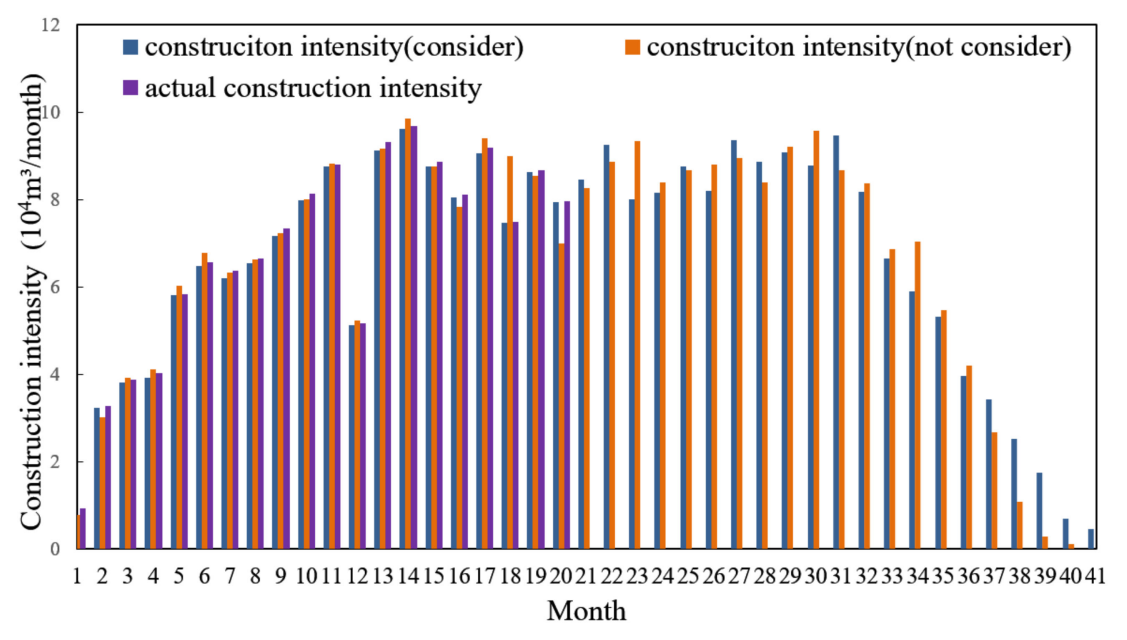

Figure 10. The comparison of the construction intensity with and without the temperature field.

\section{Conclusions}

Joint grouting simulation is an important aspect of dam construction schedule management, which has a crucial influence on the safety and construction schedule of the dam project. However, the current construction simulation model simplified the temperature factors in joint grouting simulation, which led to a deviation between the simulation schedule and the actual situation. Meanwhile, the majority of existing temperature prediction studies were based on deterministic point prediction, which cannot quantify the uncertainties of the prediction value. In order to solve these problems, this paper proposed a real-time construction simulation method coupling concrete temperature field interval prediction model. The main contributions of this paper are as follows:

1. A real-time construction simulation model was proposed. The model coupled the dam block simulation and joint grouting simulation, and analyzes the relationship between them.

2. A high-precision, high-generalization capability interval prediction model was established for concrete temperature prediction. The interval prediction model was constructed based on the hybrid-kernel relevance vector machine (HK-RVM) and the improved grasshopper optimization algorithm (IGOA). Compared with the unmodified models and the common models, the proposed model demonstrates superiority with regard to the accuracy, generalization ability.

3. A concept drift detection method based on the variable window technology was developed for updating the prediction model.

In the future, further study on the influence of the temperature field on dam construction schedules is necessary. Based on the concrete temperature field prediction model, the placing intermittent time of the concrete dam block can be optimized to realize the optimization of the entire dam construction schedule.

Author Contributions: Conceptualization, W.S.; T.G. and B.R.; data curation, W.S.; formal analysis, W.S.; methodology, W.S.; validation, W.S.; writing—original draft, W.S.; writing-review and editing, T.G.; B.R.; J.Y.; J.W. and B.W. All authors have read and agreed to the published version of the manuscript.

Funding: This research was funded by the National Key R\&D Program of China, grant number 2018YFC0406704; the National Natural Science Foundation of China of China, grant number 51839007; the Natural Science Foundation of Tianjin, grant number 19JCQNJC06800; the Natural Science Foundation of Tianjin, grant number 17JCQNJC07100.

Conflicts of Interest: The authors declare no conflict of interest. 


\section{Appendix A}

Table A1. Symbol descriptions.

\begin{tabular}{|c|c|c|c|}
\hline Symbol & Definition & Symbol & Definition \\
\hline$i$ & Dam monolith number, $i=1,2, \ldots, \mathrm{n}$ & $\Delta G(i, t)$ & $\begin{array}{l}\text { The grouting elevation variation of the } i \text { th dam } \\
\text { monolith from }(t-1) \text { to } t\end{array}$ \\
\hline$C I$ & Construction index & $\operatorname{TF}(l, t)$ & The temperature of $l$ th dam block at the time $t$ \\
\hline$H(i, 0)$ & $\begin{array}{l}\text { The pouring elevation of the } i \text { th dam } \\
\text { monolith at the time } 0\end{array}$ & $\operatorname{Age}(l, t)$ & The concrete age of $l$ th dam block at the time $t$ \\
\hline$G(i, 0)$ & $\begin{array}{l}\text { The grouting elevation of the } i \text { th dam } \\
\text { monolith at the time } 0\end{array}$ & $C W(l, t)$ & The cover weight of $l$ th dam block at the time $t$ \\
\hline$S$ & Alternative construction schemes & $T P$ & Pouring temperature \\
\hline$H_{r}(i)$ & $\begin{array}{l}\text { The pouring elevation of the } i \text { th dam } \\
\text { monolith at the time } t\end{array}$ & $T W$ & Cooling water temperature \\
\hline$H(i, t)$ & $\begin{array}{l}\text { The pouring elevation of the } i \text { th dam } \\
\text { monolith at the time } t\end{array}$ & $Q$ & Cooling water flow \\
\hline$\Delta H(i, t)$ & $\begin{array}{l}\text { The pouring elevation variation of the } i \text { th } \\
\text { dam monolith from }(t-1) \text { to } t\end{array}$ & $T_{\text {air }}$ & Ambient temperature \\
\hline$H_{\max }$ & Maximum dam height & $T N$ & The temperatures of upper and lower dam blocks \\
\hline$D(i)$ & $\begin{array}{l}\text { The placement duration of a dam block in } \\
\text { the ith dam monolith }\end{array}$ & $H K R V M$ & Hybrid-kernel relevance vector machine \\
\hline$\Psi(i)$ & $\begin{array}{l}\text { The appearance requirement of the } i \text { th dam } \\
\text { monolith }\end{array}$ & $O_{j g}$ & The output of joint grouting simulation \\
\hline$q(v)$ & The duration of the $v$ th working procedure & Time $_{\text {grout }}$ & $\begin{array}{c}\text { The grouting time of each grouting area (start } \\
\text { time) }\end{array}$ \\
\hline$O_{d b}$ & The output of dam block simulation & CTime & The current time \\
\hline$S_{\text {opt }}$ & The optimal construction scheme & Time $_{\text {pouring }}(l, 1)$ & The pouring time of $l$ th dam block \\
\hline Time $_{\text {pouring }}$ & $\begin{array}{l}\text { The pouring time of each dam block (start } \\
\text { time and end time) }\end{array}$ & $H_{\text {constrain }}$ & The constraint of cantilever height difference \\
\hline
\end{tabular}

\section{Appendix B}

Table A2. The Benchmark Functions.

\begin{tabular}{|c|c|c|c|}
\hline Function & Dim & Range & Fmin \\
\hline$F_{1}(x)=\sum_{i=1}^{n} x_{i}^{2}$ & 30 & {$[-100,100]$} & 0 \\
\hline$F_{2}(x)=\sum_{i=1}^{n}\left|x_{i}\right|+\prod_{i=1}^{n}\left|x_{i}\right|$ & 30 & {$[-10,10]$} & 0 \\
\hline$F_{3}(x)=\sum_{i=1}^{n}\left(\sum_{j=1}^{i} x_{j}\right)$ & 30 & {$[-100,100]$} & 0 \\
\hline$F_{4}(x)=\max _{i}\left\{\left|x_{i}\right|, 1 \leq i \leq n\right\}$ & 30 & {$[-100,100]$} & 0 \\
\hline$F_{5}(x)=\sum_{i=1}^{n}\left(x_{i}+0.5\right)^{2}$ & 30 & {$[-100,100]$} & 0 \\
\hline$F_{6}(x)=\sum_{i=1}^{n} i x_{i}^{4}+\operatorname{random}[0,1)$ & 30 & {$[-100,100]$} & 0 \\
\hline$F_{7}(x)=-20 \exp \left(-0.2 \sqrt{\frac{1}{n}} \sum_{i=1}^{n} x_{i}^{2}\right)-\exp \left(\frac{1}{n} \sum_{i=1}^{n} \cos \left(2 \pi x_{i}\right)\right)+20+e$ & 30 & {$[-32,32]$} & 0 \\
\hline$F_{8}(x)=\frac{1}{4000} \sum_{i=1}^{n} x_{i}^{2}-\prod_{i=1}^{n} \cos \left(\frac{x_{i}}{\sqrt{i}}\right)$ & 30 & {$[-600,600]$} & 0 \\
\hline $\begin{array}{l}F_{9}(x)=\frac{\pi}{n}\left\{10 \sin \left(\pi y_{1}\right)+\sum_{i=1}^{n-1}\left(y_{i}-1\right)^{2}\left[1+10 \sin ^{2}\left(\pi y_{i+1}\right)\right]+\left(y_{n}-1\right)^{2}\right\} \\
+\sum_{i=1}^{n} u\left(x_{i}, 10,100,4\right) \\
y_{i}=1+0.25\left(x_{i}+1\right) \\
u\left(x_{i}, a, k, m\right)= \begin{cases}k\left(x_{i}-a\right)^{m} & x_{i}>a \\
0 & -a<x_{i}<a \\
k\left(-x_{i}-a\right)^{m} & x_{i}>a\end{cases} \end{array}$ & 30 & {$[-50,50]$} & 0 \\
\hline $\begin{array}{l}F_{10}(x)=0.1\left\{\sin ^{2}\left(3 \pi x_{1}\right)+\sum_{i=1}^{n}\left(x_{i}-1\right)^{2}\left[1+\sin ^{2}\left(3 \pi x_{1}+1\right)\right]\right. \\
+\left(x_{n}-1\right)^{2}\left[1+\sin ^{2}\left(2 \pi x_{n}\right]\right\}+\sum_{i=1}^{n} u\left(x_{i}, 5,100,4\right)\end{array}$ & 30 & {$[-50,50]$} & 0 \\
\hline
\end{tabular}




\section{References}

1. Wang, R. Key Technologies in the Design and Construction of $300 \mathrm{~m}$ Ultra-High Arch Dams. Engineering 2016, 2, 350-359. [CrossRef]

2. Wei, P.; Shi, J.; Lin, P.; Qiao, Y. Study on grouting timing relating to riverbed foundation of Wudongde arch dam. In Proceedings of the 10th Asian Rock Mechanics Symposium, ARMS 2018, Singapore, 29 October-3 November 2018; International Society for Rock Mechanics: Singapore, 2018.

3. Abourizk, S.; Halpin, D.W.; Mohamed, Y.; Hermann, U. Research in Modeling and Simulation for Improving Construction Engineering Operations. J. Constr. Eng. Manag. 2011, 137, 843-852. [CrossRef]

4. Martínez, J.C. Methodology for Conducting Discrete-Event Simulation Studies in Construction Engineering and Management. J. Constr. Eng. Manag. 2010, 136, 3-16. [CrossRef]

5. Mawdesley, M.J.; Al-Jibouri, S. Modelling construction project productivity using systems dynamics approach. Int. J. Prod. Perform. Manag. 2009, 59, 18-36. [CrossRef]

6. Dabirian, S.; Khanzadi, M.; Moussazadeh, M. Predicting labor costs in construction projects using agent-based modeling and simulation. Sci. Iran. 2016, 23, 91-101. [CrossRef]

7. Moradi, S.; Nasirzadeh, F.; Golkhoo, F. A hybrid SD-DES simulation approach to model construction projects. Constr. Innov. 2015, 15, 66-83. [CrossRef]

8. Nasirzadeh, F.; Khanzadi, M.; Mir, M. A hybrid simulation framework for modelling construction projects using agent-based modelling and system dynamics: An application to model construction workers' safety behavior. Int. J. Constr. Manag. 2017, 18, 132-143. [CrossRef]

9. De Salles, D.C.; Neto, A.C.G.; Marujo, L.G. Using fuzzy logic to implement decision policies in system dynamics models. Expert Syst. Appl. 2016, 55, 172-183. [CrossRef]

10. Zhong, D.; Wu, K.; Ren, B. Dynamic simulation for high arch dam construction processes based on object-oriented technology. J. Tianjin Univ. Sci. Technol. 2007, 40, 976-982.

11. Zhong, D.-H.; Ren, B.; Wu, K. Construction simulation and real-time control for high arch dam. Trans. Tianjin Univ. 2008, 14, 248-253. [CrossRef]

12. Zhong, D.-H.; Ren, B.; Li, M.; Wu, B.; Li, M. Theory on real-time control of construction quality and progress and its application to high arc dam. Sci. China Ser. E Technol. Sci. 2010, 53, 2611-2618. [CrossRef]

13. Zhou, H.; Zhao, C.; Wang, F.; Liang, Z. Feedback Design of Temperature Control Measures for Concrete Dams based on Real-Time Temperature Monitoring and Construction Process Simulation. KSCE J. Civ. Eng. 2017, 22, 1584-1592. [CrossRef]

14. Liu, X.; Zhang, C.; Chang, X.; Zhou, W.; Cheng, Y.; Duan, Y. Precise simulation analysis of the thermal field in mass concrete with a pipe water cooling system. Appl. Therm. Eng. 2015, 78, 449-459. [CrossRef]

15. Zhu, Z.; Liu, Y.; Tan, Y. Simulation of Temperature Field of High Arch Dams Considering Solar Radiation. In Proceedings of the 6th International Conference on Environmental Science and Civil Engineering, ESCE 2020, Nanchang, China, 4-5 January 2020; Institute of Physics Publishing: Nanchang, China, 2020.

16. Nguyen, T.Q.; Vuong, L.C.; Le, C.M.; Ngo, N.K.; Nguyen-Xuan, H. A data-driven approach based on wavelet analysis and deep learning for identification of multiple-cracked beam structures under moving load. Measurement 2020, 162, 107862. [CrossRef]

17. Borate, P.; Wang, G.; Wang, Y. Data-Driven Structural Health Monitoring Approach Using Guided Lamb Wave Responses. J. Aerosp. Eng. 2020, 33, 04020033. [CrossRef]

18. Abba, S.; Usman, A.; Işik, S. Simulation for response surface in the HPLC optimization method development using artificial intelligence models: A data-driven approach. Chemom. Intell. Lab. Syst. 2020, 201, 104007. [CrossRef]

19. Han, Y.; Huang, D.; Wang, H. Research on optimization of second-stage cooling of high arch dam concrete. J. China Three Gorges Univ. 2007, 29, 109-112.

20. Fu, X.; Zhou, Y.; Huang, Y.; Zhou, S.; Li, J. BP-neural network based analysis on second-stage cooling of dam concrete. Water Resour. Hydropower Eng. 2012, 43, 50-53.

21. Zhong, D.-H.; Zhao, C.; Ren, B. Research on analysis method for temperature control information of high arch dam construction. Sci. China Ser. E Technol. Sci. 2011, 54, 40-46. [CrossRef]

22. Zhao, Y.; Tong, Y. Application of $\operatorname{MGM}(1, N)$ on Concrete Temperature Prediction of RCC Dam. In Proceedings of the 4th International Conference on Technology of Architecture and Structure (ICTAS 2011), Zurich, Switzerland, 22-24 September 2011; Trans Tech Publications Ltd.: Zurich, Switzerland, 2012; pp. $2406-2410$. 
23. Bhattarai, S.; Zhou, Y.; Zhao, C.; Zhou, H. Application of soft computing techniques for predicting cooling time required dropping initial temperature of mass concrete. Civ. Eng. J. 2017, 26, 189-203. [CrossRef]

24. Bhattarai, S.; Zhou, Y.; Zhao, C.; Zhou, H. Predicting temperature drop rate of mass concrete during an initial cooling period using genetic programming. In Proceedings of the 2017 International Conference on Sensors, Materials and Manufacturing, ICSMM 2017, Chiayi, Taiwan, 24-26 November 2017; Institute of Physics Publishing: Chiayi, Taiwan, 2018.

25. Kang, F.; Li, J.; Dai, J. Prediction of long-term temperature effect in structural health monitoring of concrete dams using support vector machines with Jaya optimizer and salp swarm algorithms. Adv. Eng. Softw. 2019, 131, 60-76. [CrossRef]

26. Chen, S.; Gu, C.; Lin, C.; Zhao, E.; Song, J. Safety Monitoring Model of a Super-High Concrete Dam by Using RBF Neural Network Coupled with Kernel Principal Component Analysis. Math. Probl. Eng. 2018, 2018, 1-13. [CrossRef]

27. Adjenughwure, K.; Papadopoulos, B. Fuzzy-statistical prediction intervals from crisp regression models. Evol. Syst. 2019, 11, 201-213. [CrossRef]

28. Kodaira, D.; Jung, W.; Han, S. Optimal Energy Storage System Operation for Peak Reduction in a Distribution Network Using a Prediction Interval. IEEE Trans. Smart Grid 2020, 11, 2208-2217. [CrossRef]

29. Zhang, C.; Zhao, Y.; Fan, C.; Li, T.; Zhang, X.; Li, J. A generic prediction interval estimation method for quantifying the uncertainties in ultra-short-term building cooling load prediction. Appl. Therm. Eng. 2020, 173, 115261. [CrossRef]

30. Zhu, X.; Zeng, B.; Dong, H.; Liu, J. An interval-prediction based robust optimization approach for energy-hub operation scheduling considering flexible ramping products. Energy 2020, 194, 116821. [CrossRef]

31. Hu, J.; Lin, Y.; Tang, J.; Zhao, J. A new wind power interval prediction approach based on reservoir computing and a quality-driven loss function. Appl. Soft Comput. 2020, 92, 106327. [CrossRef]

32. Yang, X.; Fu, G.; Zhang, Y.; Kang, N.; Gao, F. A Naive Bayesian Wind Power Interval Prediction Approach Based on Rough Set Attribute Reduction and Weight Optimization. Energies 2017, 10, 1903. [CrossRef]

33. Chen, L.; Liu, Y.; Zhao, J.; Wang, W.; Liu, Q. Prediction intervals for industrial data with incomplete input using kernel-based dynamic Bayesian networks. Artif. Intell. Rev. 2016, 46, 307-326. [CrossRef]

34. Song, L.; Minku, L.L.; Yao, X. Software Effort Interval Prediction via Bayesian Inference and Synthetic Bootstrap Resampling. ACM Trans. Softw. Eng. Methodol. 2019, 28, 1-46. [CrossRef]

35. Pokhriyal, H.; Balakrishna, N. Bootstrap prediction intervals for autoregressive conditional duration models. J. Stat. Comput. Simul. 2019, 89, 2930-2950. [CrossRef]

36. Beyaztas, U.; Arikan, B.B.; Beyaztas, B.H.; Kahya, E. Construction of prediction intervals for Palmer Drought Severity Index using bootstrap. J. Hydrol. 2018, 559, 461-470. [CrossRef]

37. Zhang, G.; Allaire, D.; McAdams, D.A.; Shankar, V. Generating Technology Evolution Prediction Intervals Using a Bootstrap Method. J. Mech. Des. 2019, 141, 061401. [CrossRef]

38. Zhang, G.; Li, Z.; Zhang, K.; Zhang, L.; Hua, X.; Wang, Y. Multi-objective interval prediction of wind power based on conditional copula function. J. Mod. Power Syst. Clean Energy 2019, 7, 802-812. [CrossRef]

39. Pang, J.; Liu, D.; Peng, Y.; Peng, X. Optimize the Coverage Probability of Prediction Interval for Anomaly Detection of Sensor-Based Monitoring Series. Sensors 2018, 18, 967. [CrossRef]

40. Liu, Y.; Yan, J.; Han, S.; David, I.; Tian, D.; Gao, L. An optimized short-term wind power interval prediction method considering NWP accuracy. Chin. Sci. Bull. 2014, 59, 1167-1175. [CrossRef]

41. Sachindra, D.; Ahmed, K.; Rashid, M.; Shahid, S.; Perera, B. Statistical downscaling of precipitation using machine learning techniques. Atmospheric Res. 2018, 212, 240-258. [CrossRef]

42. Gonen, M.; Alpaydin, E. Multiple kernel learning algorithms. J. Mach. Learn. Res. 2011, 12, 2211-2268.

43. Guo, S.; Zhao, H.; Zhao, H. A New Hybrid Wind Power Forecaster Using the Beveridge-Nelson Decomposition Method and a Relevance Vector Machine Optimized by the Ant Lion Optimizer. Energies 2017, 10, 922. [CrossRef]

44. Qiao, W.; Huang, K.; Azimi, M.; Han, S. A Novel Hybrid Prediction Model for Hourly Gas Consumption in Supply Side Based on Improved Whale Optimization Algorithm and Relevance Vector Machine. IEEE Access 2019, 7, 88218-88230. [CrossRef]

45. Bui, D.T.; Shahabi, H.; Shirzadi, A.; Chapi, K.; Hoang, N.-D.; Pham, B.T.; Bui, Q.-T.; Tran, C.-T.; Panahi, M.; Ahmed, B.; et al. A Novel Integrated Approach of Relevance Vector Machine Optimized by Imperialist Competitive Algorithm for Spatial Modeling of Shallow Landslides. Remote Sens. 2018, 10, 1538. [CrossRef] 
46. Nhat Duc, H.; Dieu Tien, B. A novel relevance vector machine classifier with Cuckoo search optimization for spatial prediction of landslides. J. Comput. Civ. Eng. 2016, 30, 04016001.

47. Xiang, Q.; Yuan, Y.; Yu, Y.; Chen, K. Rotor Position Self-Sensing of SRM Using PSO-RVM. Energies 2018, 11, 66. [CrossRef]

48. Jiang, Y.; Xu, Z.; Zhang, Z.; Liu, X. A novel shearer cutting pattern recognition model with chaotic gravitational search optimization. Measurement 2019, 144, 225-233. [CrossRef]

49. Lou, J.; Jiang, Y.; Shen, Q.; Wang, R. Failure prediction by relevance vector regression with improved quantum-inspired gravitational search. J. Netw. Comput. Appl. 2018, 103, 171-177. [CrossRef]

50. Saremi, S.; Mirjalili, S.; Lewis, A. Grasshopper Optimisation Algorithm: Theory and application. Adv. Eng. Softw. 2017, 105, 30-47. [CrossRef]

51. Heidari, A.A.; Faris, H.; Aljarah, I.; Mirjalili, S. An efficient hybrid multilayer perceptron neural network with grasshopper optimization. Soft Comput. 2018, 23, 7941-7958. [CrossRef]

52. Gama, J.; Žliobaitè, I.; Bifet, A.; Pechenizkiy, M.; Bouchachia, A. A survey on concept drift adaptation. ACM Comput. Surv. 2014, 46, 1-37. [CrossRef]

53. Wares, S.; Isaacs, J.P.; Elyan, E. Data stream mining: Methods and challenges for handling concept drift. SN Appl. Sci. 2019, 1, 1412. [CrossRef]

54. Liu, G.; Cheng, H.; Qin, Z.; Liu, Q.; Liu, C. E-CVFDT: An improving CVFDT method for concept drift data stream. In Proceedings of the 2013 International Conference on Communications, Circuits and Systems, ICCCAS 2013, Chengdu, China, 15-17 November 2013; IEEE Computer Society: Chengdu, China, 2013; pp. 315-318.

55. Bifet, A.; Gavalda, R. Learning from time-changing data with adaptive windowing. In Proceedings of the 7th SIAM International Conference on Data Mining, Minneapolis, MN, USA, 26-28 April 2007; Society for Industrial and Applied Mathematics Publications: Minneapolis, MN, USA, 2007; pp. 443-448.

56. Li, R.; Jin, Y. A wind speed interval prediction system based on multi-objective optimization for machine learning method. Appl. Energy 2018, 228, 2207-2220. [CrossRef]

(C) 2020 by the authors. Licensee MDPI, Basel, Switzerland. This article is an open access article distributed under the terms and conditions of the Creative Commons Attribution (CC BY) license (http://creativecommons.org/licenses/by/4.0/). 